\title{
Importance of brain-type fatty acid binding protein for cell-biological processes in human renal carcinoma cells
}

\author{
ANGELIKA TÖLLE ${ }^{1}$, HANS KRAUSE $^{1}$, KURT MILLER ${ }^{1}$, KLAUS JUNG $^{2}$ and CARSTEN STEPHAN ${ }^{1,2}$ \\ ${ }^{1}$ Department of Urology, Charité-Universitätsmedizin Berlin; ${ }^{2}$ Berlin Institute for \\ Urologic Research, Charitéplatz 1, D-10117 Berlin, Germany
}

Received October 27, 2010; Accepted December 13, 2010

DOI: $10.3892 /$ or.2011.1209

\begin{abstract}
The molecular mechanisms underlying renal cell carcinoma (RCC) development and progression are still not completely understood. The importance of fatty acid binding proteins (FABP) for the progression of carcinomas has been shown for several tumors. However, the importance of braintype FABP (B-FABP) in cell-biological processes in renal carcinoma cells is unknown. Therefore, it was the aim of this study to evaluate the role of B-FABP in processes such as proliferation, migration and invasion. By using the approach of down- and up-regulation of B-FABP in human kidney carcinoma cells Caki-2 and Caki-1, the potential participation of B-FABP in proliferation, migration and invasion was demonstrated. B-FABP was down-regulated at both mRNA and protein levels following treatment of Caki-2 cells with B-FABP siRNA. Down-regulation of B-FABP decreased cell proliferation and migration but did not affect invasion. The transfection of Caki-1 cells with human B-FABP cDNA generated an increment of B-FABP mRNA but the protein was not detectable. Transfected Caki-1 cells developed a faster proliferation compared to untreated cells. An effect on the process of invasion was not observed. Our data suggest that B-FABP is involved in cell proliferation and migration of human renal carcinoma cells. The detailed molecular mechanisms remain to be elucidated.
\end{abstract}

\section{Introduction}

Brain-type fatty acid binding protein (B-FABP) is a member of a family of cytosolic protein, which binds intracellular hydrophobic ligands. This family contains 9 different types of fatty acid binding proteins (FABP). Their expression occurs in a regulated and tissue-specific manner. So FABPs are involved in transporting fatty acids to cellular compartments, modulating intracellular lipid metabolism and regulating gene

Correspondence to: Dr Angelika Tölle, Department of Urology, Charité-Universitätsmedizin Berlin, Charitéplatz 1, D-10117 Berlin, Germany

E-mail: angelika.toelle@charite.de

Key words: brain-type fatty acid binding protein, renal carcinoma cells, proliferation, migration expression (1). The importance of FABPs for the progression of carcinomas was shown for prostate (2), breast (3) and bladder cancer (4) as well as for astrocytomas (5). Recently, a significant association of adipocyte-type FABP (A-FABP) with breast cancer risk was shown (6). Furthermore, an increased expression of B-FABP was reported in clear cell renal cell carcinomas (RCC) (7).

Cell culture systems are particularly suited to study the effect of FABPs on cell-biological processes such as proliferation, migration and invasion. For example, the human breast cancer cell line MCF7 does not express heart-type fatty acid binding protein (H-FABP), whereas these cells transfected with the bovine H-FABP are characterized by growth inhibition and lower tumorigenicity in nude mice (8). An increased uptake of fatty acids was observed by MCF7 cells transfected with H-FABP in comparison with native MCF7 cells (9). Moreover, epidermal-type fatty acid binding protein (E-FABP) promotes cell proliferation and invasion in oral squamous cell carcinoma (10). Melanoma cells express B-FABP and a relationship to proliferation and invasion was shown (11). However, the importance of B-FABP in the decisive cell-biological processes in renal carcinoma cells is unknown. Therefore, it was the aim of this study to evaluate the role of B-FABP in these processes of proliferation, migration and invasion. By using the approach of down- and up-regulation of B-FABP in human kidney carcinoma cells Caki-1 and Caki-2, the potential participation of B-FABP in these processes is demonstrated.

\section{Materials and methods}

Cell culture. Human kidney carcinoma cell line Caki-2 and Caki-1 (obtained from American Type Culture Collection, Manassas, VA) were cultured in 90\% McCoy's 5A medium (Invitrogen $\mathrm{GmbH}$, Karlsruhe, Germany), supplemented with $10 \%$ fetal calf serum (PAA Laboratories GmbH, Pasching, Austria) and antibiotics [1x penicillin/streptomycin: $100 \mathrm{U} / \mathrm{ml}$ and $100 \mu \mathrm{g} / \mathrm{ml}$, (PAA Laboratories)]. Cells were incubated in a humidified atmosphere containing $5 \% \mathrm{CO}_{2}$ at $37^{\circ} \mathrm{C}$. The Caki-2 cell line established from primary renal carcinoma of a 69-year-old Caucasian man, expresses B-FABP. In contrast, the Caki-1 cell line originated from a metastatic site of a renal clear cell carcinoma of a 49-year-old Caucasian man expresses B-FABP in a very low quantity. 
Stable shRNA and cDNA transfection. A HuSh 29mer shRNA construct against B-FABP (OriGene Technologies, Inc., Rockville, USA) was used to silence B-FABP expression in Caki-2 cells. The vector pRS contains a puromycin resistance gene and a 29 nucleotide gene specific sequence insert. A pRS vector without shRNA cassette insert served as control. FuGENE ${ }^{\circledR}$ HD (Roche Applied Science, Mannheim, Germany) was used as transfection reagent. Transfection conditions were optimized initially. The ratio of the transfection reagent $(\mu \mathrm{l})$ to DNA $(\mu \mathrm{g})$ ratio was $3: 1$. The transfection reagent complex, FuGENE ${ }^{\circledR}$ HD and specific siRNA (AACTGTAAGTCTGTTGTTAGCCTGGATGG) as well as the control siRNA, were prepared according to the manufacturer's protocol. The complex was incubated for $15 \mathrm{~min}$ at room temperature for the silence experiment and the Caki- 2 cells were subsequently transfected. The cells were plated at $2.5 \times 10^{5}$ in $2 \mathrm{ml}$ into a 6 -well plate in an antibiotic-free medium with $10 \%$ FCS. When the cells reached $80 \%$ confluence, they were ready for transfection. Afterwards, the growth medium was replaced by serum-free medium and $100 \mu l$ of the transfection complex were added below the surface of the medium. Following the transfection, the Caki- 2 cells were incubated for $24 \mathrm{~h}$ and the medium was replaced by medium with $10 \%$ FCS without any antibiotics. After an additional incubation of $24 \mathrm{~h}$, puromycin $(3 \mu \mathrm{g} / \mathrm{ml})$ was added for selecting of transfected cells.

The conditions for true-transfection in Caki-1 cells were slightly different. A TrueORF vector system with a B-FABP sequence (OriGene Technologies) was used for overexpression experiments. The vector pCMV6-AC-GFP contains a geneticin resistance gene and a clone of human B-FABP. A pCMV6-AC-GFP vector without cDNA clone served as control. The true-transfection complex was incubated $20 \mathrm{~min}$ and then added to Caki- 1 cells. The cells were plated at $6 \times 10^{4}$ in $1 \mathrm{ml}$ into a 24-well plate in an antibiotic-free medium with $10 \%$ FCS. Only $25 \mu 1$ of the transfection complex were added to the medium. The Caki-1 cells were incubated for $24 \mathrm{~h}$ and then the medium was replaced by medium with $10 \%$ FCS and geneticin G418 (1 mg/ml) for selecting of transfected cells.

In both experiments the uninfected cells were killed within one week and the clone population started to grow. Specific silence of the targeted gene and the expression of B-FABP were confirmed by reverse transcription-quantitative polymerase chain reaction (RT-qPCR) analysis.

RT-qPCR analysis. For RT-qPCR analysis of B-FABP mRNA levels, total RNA was isolated from transfected Caki-2 cells using the RNeasy Mini Kit (Qiagen GmbH, Hilden, Germany) according to the manufacturer's instructions including an additional genomic DNA digestion step with DNase I. The RNA yield and quality were determined by using the NanoDrop ${ }^{\circledR}$ ND-1000 Spectrophotometer (NanoDrop Technologies, Montchanin, USA) and Agilent 2100 Bioanalyzer (Agilent Technologies, Waldbronn, Germany). RNA (1 $\mu \mathrm{g})$ was reverse transcribed using the Transcriptor First Strand cDNA Synthesis Kit (Roche Applied Science) by random hexamer priming method according to the manufacturer's recommendations. RT-qPCR was performed with the LigthCycler ${ }^{\circledR} 480$ Instrument (Roche Applied Science) equipped with a 96-well block. The B-FABP (accession no.: NM_001446) specific
PCR assay was designed with the web-based ProbeFinder software Version 2.40 for Human (www.universalprobelibrary. com) using primers and probe of the Universal ProbeLibrary (UPL; Roche Applied Science). We selected for the B-FABP RT-qPCR the UPL assay with following primer-probe combination: the forward primer 5'-ctcagcacattcaagaacacg-3' and the reverse primer 5 '-ccatccaggctaacaacagac-3' with the UPL probe no. 33 (Roche cat. no. 04687663001). The detailed conditions for PCR were previously described (7). The peptidylproline isomerase A (PPIA) was determined as internal control for normalizing cellular mRNA data. The intraserial run precision of the PCR measurements amounted to a variation coefficient of $0.52 \%$ at a mean Cq-value of 26.68 for B-FABP and of $0.56 \%$ at a mean Cq-value of 22.83 for PPIA, respectively.

Immunoblotting. All cells were lysed in ice-cold NP-40 lysis buffer consisting of $1 \% \mathrm{NP}-40,150 \mathrm{mM} \mathrm{NaCl}, 50 \mathrm{mM}$ Tris- $\mathrm{HCl}, \mathrm{pH} 8.0,0.5 \%$ Na-deoxycholate, $0.1 \%$ SDS, $0.1 \mathrm{mM}$ phenylmethylsulfonyl fluoride, $1 \mu \mathrm{g} / \mathrm{ml}$ aprotinin and $10 \mu \mathrm{g} / \mathrm{ml}$ soybean trypsin inhibitor. Protein quantification was done by Bradford analysis. Cell extracts were analysed for B-FABP expression by separation of $40 \mu \mathrm{g}$ of protein on a 15\% SDS-polyacrylamide gel and then transferred onto a polyvinylidene fluoride membrane (Millipore, Billerica, MA, USA). B-FABP was detected with polyclonal rabbit antihuman B-FABP antibody $(1 \mu \mathrm{g} / \mathrm{ml}$; Hycult Biotechnology b.v., Uden, The Netherlands). Horseradish peroxidase-conjugated goat anti-rabbit IgG (DakoCytomation, Glostrup, Denmark) was used as secondary antibody. Actin (anti- $\beta$-actin clone AC-74, Sigma-Aldrich Chemie GmbH, Munich, Germany) served as loading control and secondary antibody was horseradish peroxidase-conjugated rabbit anti-mouse IgG (DakoCytomation). The antigen-antibody reaction was visualized by ECL Advance ${ }^{\mathrm{TM}}$ Western Blotting Detection Kit (GE Healthcare UK Limited, Little Chalfont, Buckinghamshire, UK). Intensity of the detected signals by Western blotting was quantified with Fluor-S MultiImager (Bio-Rad Laboratories, Hercules, USA).

Cell proliferation assay. The XTT-Cell Proliferation Kit (Roche Applied Science) was used to evaluate cell proliferation according to the supplier's protocol. Caki-2, Caki-1 cells as well as shRNA transfected and B-FABP transfected cells in $100 \mu \mathrm{l}$ McCoy's 5A medium were seeded at a concentration of $5 \times 10^{2}$ cells/well of 96-well culture plates. After 24, 48, 72 and $96 \mathrm{~h}$ of cultivation, $50 \mu \mathrm{l}$ of the XTT labeling mixture were added and incubated for $4 \mathrm{~h}$ in a humidified atmosphere with $5 \% \mathrm{CO}_{2}$ at $37^{\circ} \mathrm{C}$. The orange formazan formation was quantified at $492 \mathrm{~nm}$ and the reference wavelength of $690 \mathrm{~nm}$. Each experiment was done in triplicate.

Migration and matrigel invasion assay. A filter assay was employed in cell migration and invasion studies. This assay used a two-compartment system where cells may be induced to migrate from an upper compartment through a porous membrane into a lower compartment, thus following the gradient of a chemoatractant. Inserts with $8 \mu \mathrm{m}$ pore size PET membrane coated with Matrigel for invasion studies and without Matrigel for migration studies (Becton-Dickinson 

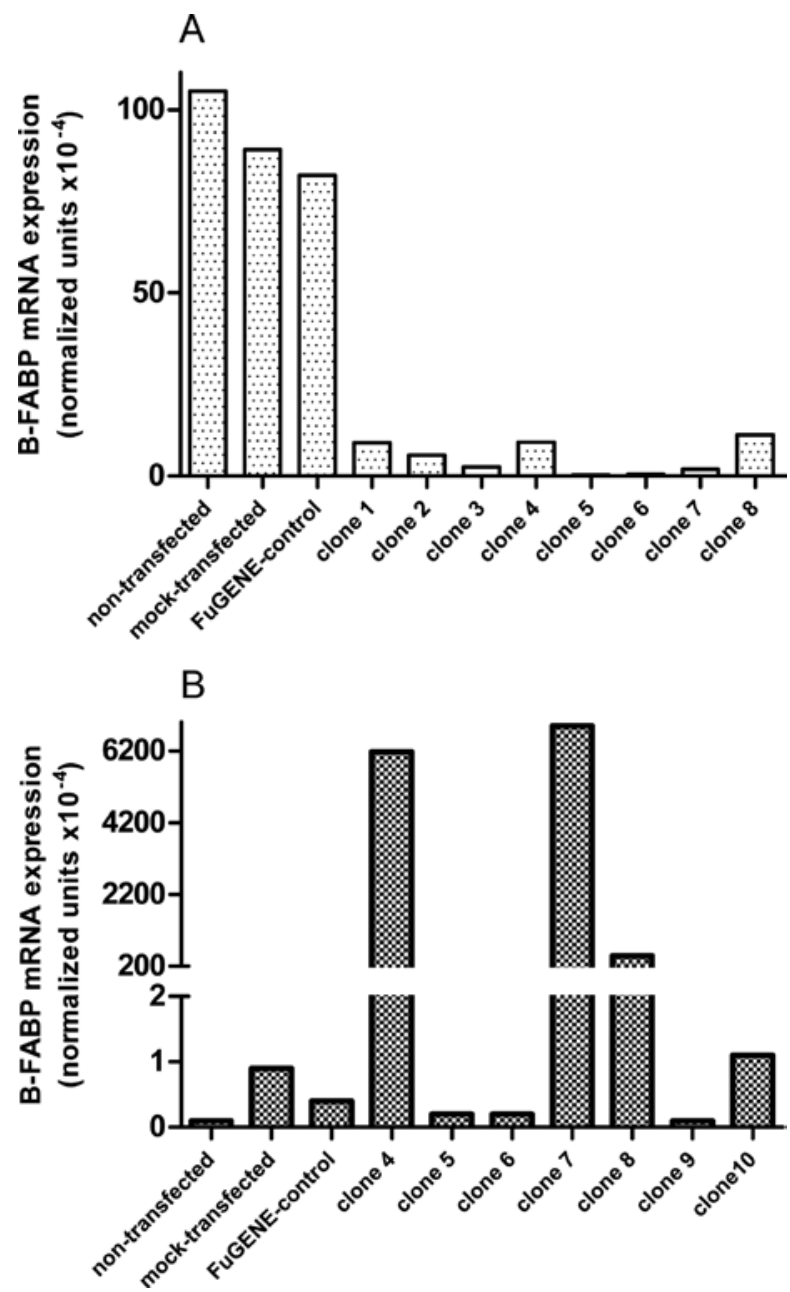

Figure 1. Quantification of B-FABP mRNA. Expression data were normalized against reference gene PPIA. (A) Caki-2 cells after siRNA transfection, (B) Caki-1 cells after true transfection.

Biosciences, Heidelberg, Germany) were placed in a 24 -well companion plate. For the migration assay, $5 \times 10^{4}$ cells seeded on the upper chamber in serum-free medium may be induced to actively migrate through the membrane onto the lower chamber with medium containing serum. The cell culture plates were incubated for $24 \mathrm{~h}$ in an incubator at $37^{\circ} \mathrm{C}$ and $5 \% \mathrm{CO}_{2}$. After this time the culture medium was removed and replaced by $450 \mu \mathrm{l}$ serum-free medium with $8 \mu \mathrm{M}$ Calcein-AM (Invitrogen $\mathrm{GmbH}$ ) followed by an incubation time of $45 \mathrm{~min}$ at $37^{\circ} \mathrm{C}$ and $5 \% \mathrm{CO}_{2}$. Afterwards, the medium was removed from the cell culture inserts and the inserts were transferred into a freshly prepared 24 -well plate containing $500 \mu \mathrm{l}$ prewarmed Trypsin/EDTA per well. The inserts were removed after an incubation time of $10 \mathrm{~min}$ at $37^{\circ} \mathrm{C}$ and $5 \%$ $\mathrm{CO}_{2}$. Finally, the Trypsin/EDTA solution containing the migratory cells and $200 \mu \mathrm{l}$ of this solution were transferred into a black flat bottom 96-well Cliniplate (Labsystems Oy, Helsinki, Finland).

For the invasion assay, $2.5 \times 10^{4}$ cells were added to the upper chamber. Plates were incubated at $37^{\circ} \mathrm{C}$ and $5 \% \mathrm{CO}_{2}$ for $22 \mathrm{~h}$. Subsequently, the inserts were transferred into a new 24-well plate containing $500 \mu \mathrm{l} /$ well of $4 \mu \mathrm{M}$ Calcein-AM followed by an incubation time of $1 \mathrm{~h}$ at $37^{\circ} \mathrm{C}$ and $5 \% \mathrm{CO}_{2}$.
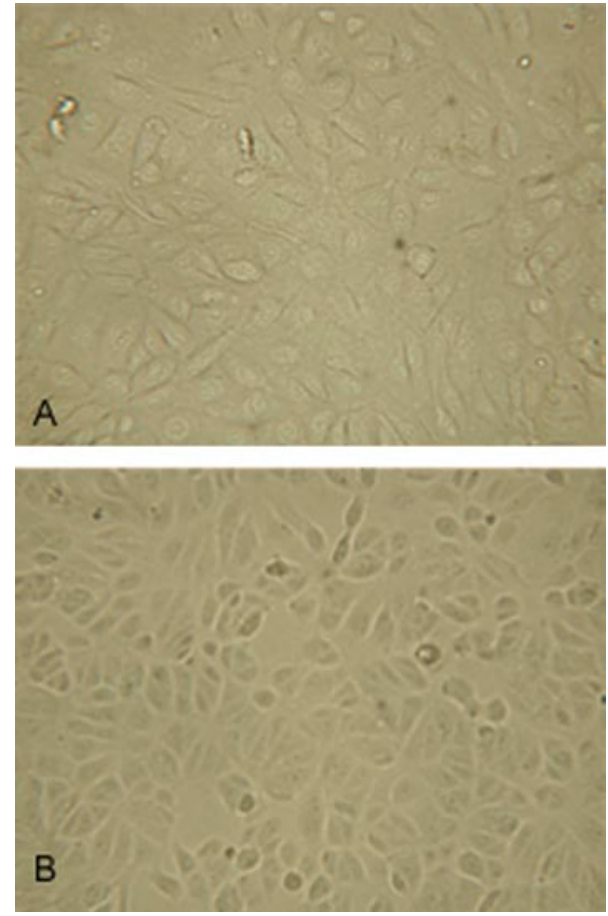

Figure 2. Microscope micrographs of Caki-2 cells and their clones. (A) untreated Caki-2 cells, (B) Caki-2 clone, objective x10.

The fluorescence of migrated and invading cells was measured at excitation/emission wavelengths of $485 / 538 \mathrm{~nm}$. The migration and invasion of the selected clones were compared with the corresponding reference of Caki-1 and Caki-2 cells, respectively. Each experiment was done in triplicate.

\section{Results}

Transfection of Caki-2 and Caki-1 cells and clonal variation of $B-F A B P$ mRNA expression. After puromycin or geneticin selection, respectively numerous clones developed. Selection for resistance to both antibiotics was an indirect test checking the stable integration of the constructs in the genome. The specific silence of the targeted gene and the implementation of B-FABP were confirmed by RT-qPCR analysis. The RNA purity was given by an absorbance $260 / 280 \mathrm{~nm}$ ratio in range over all RNA samples from 1.9 to 2.0. The RNA integrity was characterized by the RIN values from 9.8 to 10.0.

The treatment of cells with FuGENE ${ }^{\circledR}$ HD showed no influence of the B-FABP mRNA expression. The mocktransfected cells developed a similar expression level of B-FABP mRNA as the non-transfected Caki-2 and Caki-1 cells, respectively. The selected clones were characterized by different reduction and increment of B-FABP mRNA expression. Fig. 1 shows the eight Caki-2 clones with a reduced and three out of seven Caki-1 clones with an increased B-FABP mRNA. Further characterization of the transfected cells was performed with the clones 3 and 5 of the Caki- 2 cells and with the clone 7 originated from Caki-1 cells.

Proliferation and B-FABP expression. Caki-1 and Caki-2 cells are characterized by different growth properties. The 

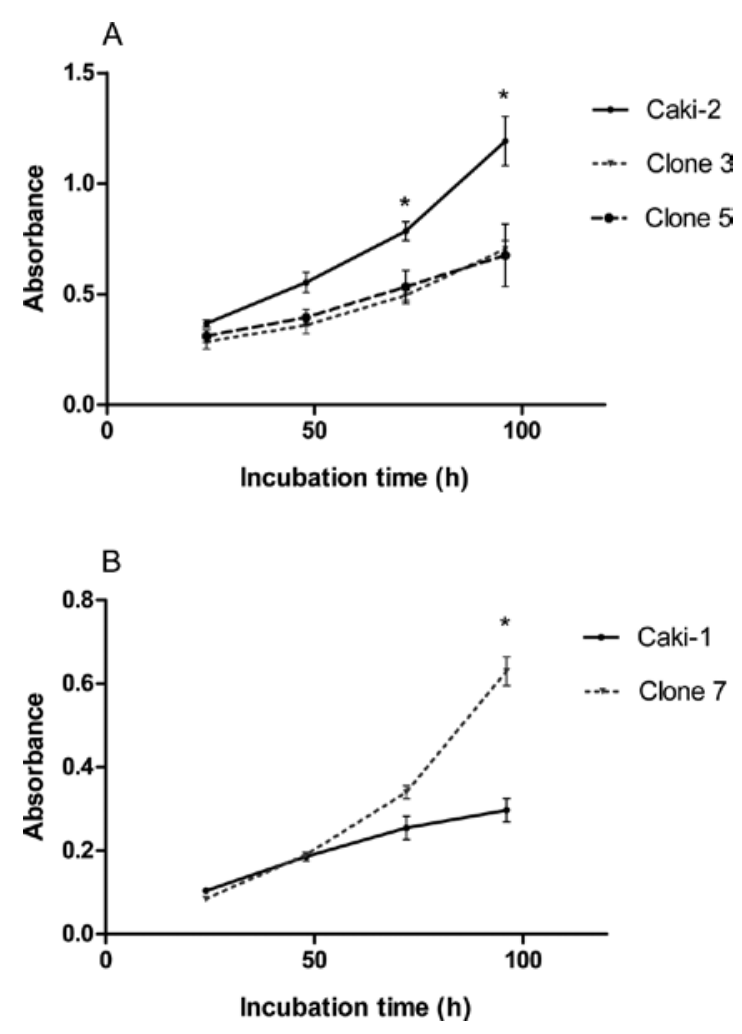

Figure 3. Proliferation of Caki cells in comparison to the transfected clones. Values are arithmetic means of three independent experiments. (A) Caki-2 cells and their clones 3 and 5 . The differences were significant for clone 3 after $48 \mathrm{~h}(\mathrm{P}=0.026), 72 \mathrm{~h}(\mathrm{P}=0.003), 96 \mathrm{~h}(\mathrm{P}=0.016)$ and for clone 5 after $72 \mathrm{~h}(\mathrm{P}=0.026), 96 \mathrm{~h}(\mathrm{P}=0.033)$. (B) Caki-1 cells and their clone 7. The differences were significant after $96 \mathrm{~h}(\mathrm{P}=0.002)$.

subcultivation ratio was 1:2 to 1:4 for Caki-1 and 1:3 to 1:6 for Caki-2 cells. Both cell lines grow adherent as monolayer. These cells show a typical epithelial-like morphology. The cells with a reduced or an increased B-FABP mRNA expression retained their original morphology compared with the untreated cell line (Fig. 2).

The proliferation of the two clones 3 and 5 of Caki- 2 cells significantly slowed down in comparison to the untreated cells (Fig. 3A). The clone 7 of Caki-1 cells proliferated faster in comparison to the untreated cells (Fig. 3B).

Clonal protein variation was monitored by immunoblotting of untreated and transfected cells. The silence of the target gene B-FABP caused a reduced protein level for both clones 3 (by $22 \%$ ) and 5 (by 11\%) compared with the untreated Caki-2 cells. The protein of B-FABP was not detectable by immunoblotting in clone 7 of Caki-1 cells transfected with human B-FABP cDNA. However, a strong signal was observed at about 30 and $45 \mathrm{kDa}$ suggesting the existence of polymers of B-FABP in transfected cells (Fig. 4).

Migration and invasion. The initial observation was that untreated Caki-2 and Caki-1 cells showed different migration and invasion properties. The migration and invasion of Caki-2 cells were slower in comparison to the Caki-1 cells. The silence of the target gene B-FABP caused a reduced migration for both clones $3(\mathrm{P}=0.008)$ and $5(\mathrm{P}=0.002)$, but unchanged matrigel invasion compared with the untreated Caki-2 cells

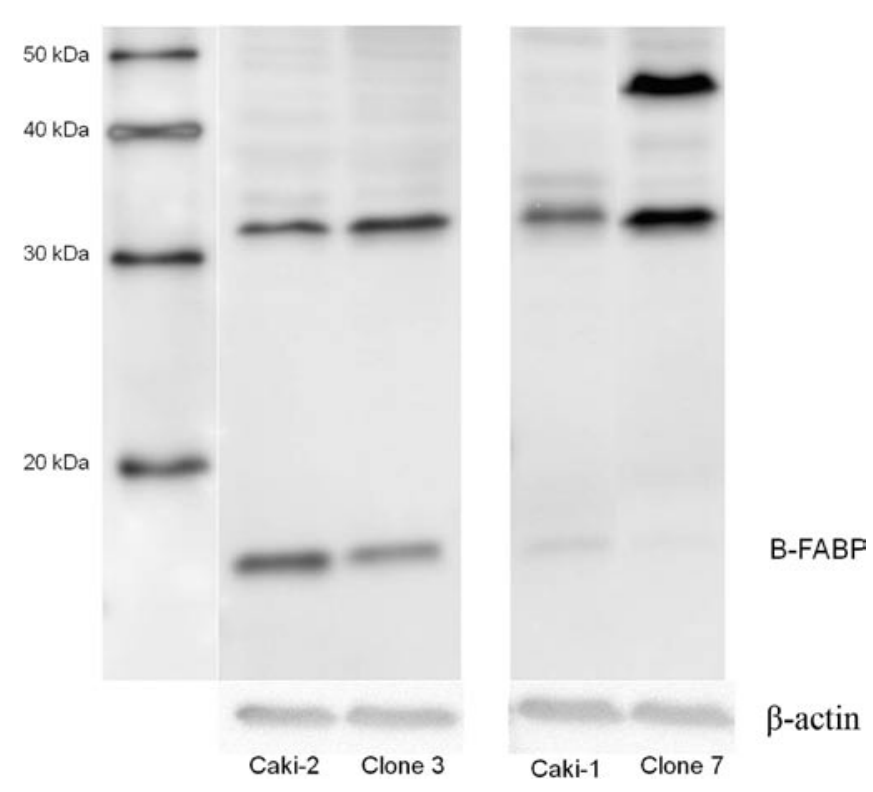

Figure 4. Immunodetection of B-FABP after transfection experiments, with B-actin as loading control. Down-regulation of B-FABP in Caki-2 cells after transfection with B-FABP siRNA and B-FABP in Caki-1 cells after transfection with human cDNA.
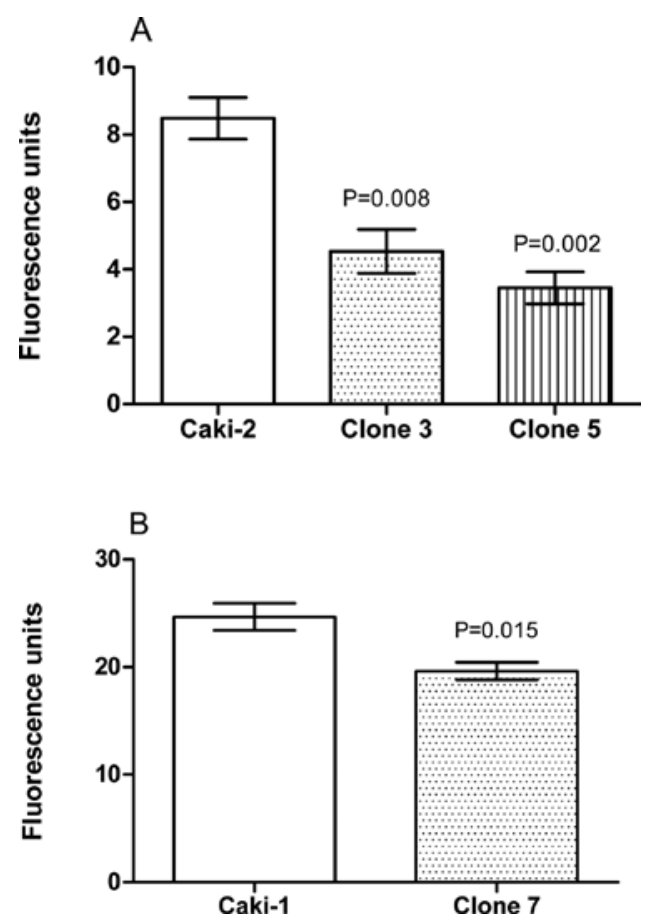

Figure 5. Migration of transfected clones in comparison to untreated Caki cells. Values are arithmetic means of three independent experiments. (A) Caki-2 cells and their clones 3 and 5 . The differences were significant for clone $3(\mathrm{P}=0.008)$ and for clone $5(\mathrm{P}=0.002)$. (B) Caki-1 cells and their clone 7 . The difference was significant $(\mathrm{P}=0.015)$.

(Figs. 5A and 6A). The migration properties of clone 7 of Caki-1 cells with an increased B-FABP mRNA expression, but an unchanged protein level was also reduced $(\mathrm{P}=0.015)$ in comparison to the untreated cells (Fig. 5B), while the invasion capacity was not changed (Fig. 6B). 

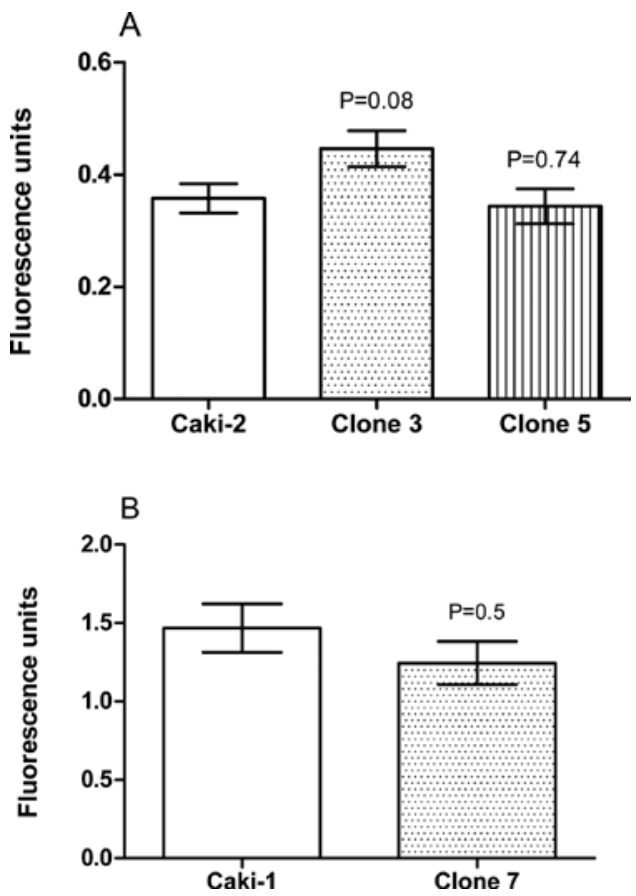

Figure 6. Invasion of transfected clones in comparison to untreated Caki cells. Values are arithmetic means of three independent experiments. (A) Caki-2 cells and their clones 3 and 5. The differences were not significant (B) Caki-1 cells and their clone 7. The difference was not significant.

\section{Discussion}

Previous studies revealed that B-FABP was overexpressed in renal cell carcinoma tissues $(7,12,13)$. The present study proves that $\mathrm{B}-\mathrm{FABP}$ influences the proliferation and migration in renal cell carcinoma cell lines whereas the invasion ability of the cells did not depend on their B-FABP content. Additionally, it is remarkable that the morphology of the transfected cells was not changed in comparison to the native cells. Until now, these effects of B-FABP on decisive cellbiologic properties were not described. Other reports provided evidence of altered fatty acid metabolism in RCC since the preferred liver-type fatty acid binding protein (L-FABP) localized in the proximal tubules of the normal kidney (14) was substituted by B-FABP in renal carcinomas (7).

The human kidney carcinoma cell line Caki-2 used in our experiments was established from primary renal carcinoma and the Caki-1 cell line originated from a metastatic site of a renal clear cell carcinoma. Both cell lines demonstrate a clear difference in B-FABP expression. In Caki-1 cells, the expression of B-FABP mRNA and protein is very low in comparison to Caki-2 cells. These results correspond to B-FABP mRNA expression data observed in human renal cell carcinoma tissue samples with increased B-FABP mRNA in malignant vs. non-malignant tissue and in dependency of the B-FABP expression on histological differentiation grade (7). Tumors with a low grading $(\mathrm{G} 1+\mathrm{G} 2)$ showed a higher B-FABP expression compared with high grading (G3+G4) and more aggressive tumors (7). Thus, the characteristic increased B-FABP mRNA expression in G1/G2 tumors in comparison to their adjacent non-malignant tissue was distinctly reduced in G3/G4 tumors like in Caki-1 cells.
The migration and invasion properties of both Caki-cell lines are different. The migration and invasion of Caki-2 cells were slower in comparison to the Caki-1 cells. These differences were the reason for performing experiments with down- and up-regulation of B-FABP to clarify its role in RCC. The proliferation and migration in Caki-2 cells with an increased content of B-FABP were notably inhibited by downregulating the $\mathrm{B}-\mathrm{FABP}$ expression with $\mathrm{B}-\mathrm{FABP}$ specific small interfering RNAs, whereas the invasion was not influenced by the $\mathrm{B}-\mathrm{FABP}$ reduction. The small decrease in the protein level by about $20 \%$ may have been insufficient to effectively change the invasion. In support of our results, both Goto et al (11) and Slipicevic et al (15) showed that proliferation and invasion of melanoma cell lines are decreased upon down-regulation of B-FABP. Similarly, Mita et al (5) showed decreased migration and invasion of B-FABP positive malignant glioma cells following B-FABP depletion. However, in contrast to our results in kidney carcinoma cells the author did not observe any effect on the proliferation when the B-FABP content was reduced in glioma cells. Other studies also proved the influence of other FABP subtypes on the proliferation in various other cells. The ability to promote cell growth by increased A-FABP in L6 myoblasts (16) E-FABP in prostate carcinoma PC-3 cells (17) as well as in oral squamous cell carcinoma cells (10) and L-FABP in hepatoma cells was shown (18).

The results obtained with $\mathrm{B}-\mathrm{FABP}$ reduction were partly supported by the inverse experiments when B-FABP was introduced in the Caki-1 cells characterized by a low B-FABP expression. We found an increased proliferation rate, unchanged invasion, but decreased migration of these transfectants. While we verified a moderate decreased B-FABP protein in the transfected Caki- 2 clones along with reduced B-FABP mRNA, this relationship was missing in the clone 7 with increased B-FABP mRNA of the Caki-1 cells. The strong increment of B-FABP mRNA in these transfected cells was not associated with a corresponding expression of active B-FABP protein with $15 \mathrm{kDa}$ molecular weight (Fig. 4). It can be assumed that the polymers of B-FABP with a molecular weight of 30 and $45 \mathrm{kDa}$ were precursor forms of the active protein. Perhaps other cellular processes were responsible for the reduced migration. Another reason might be that these cells apparently do not longer have the capability to generate B-FABP protein, because already the Caki-1 cells originated from a metastatic site of a renal clear cell carcinoma lost this attribute. It cannot be excluded that these peculiarities could also explain the described non-corresponding finding.

The molecular mechanisms of B-FABP changes in RCC remain, despite the evident cell-biological effects, to be elucidated. Until now, only in the context with the development of the CNS and with glioblastomas, the participation of B-FABP on molecular regulatory mechanisms has been shown. During CNS development of mice, B-FABP was identified as a direct target of Notch signaling in radial glia (19). It was shown in malignant glioma cells that the B-FABP expression is determined by the extent of nuclear factor 1 phosphorylation which, in turn, is controlled by a phosphatase activity specific to B-FABP positive lines $(20,21)$. It was also demonstrated for that EGFR activation induces B-FABP nuclear translocation $(22,23)$. Due to this interaction with nuclear receptors the FABP family is able to modulate the transcription (24). 
In conclusion the presence of B-FABP in renal carcinoma cell lines influence the proliferation and migration of these cells. Thus, the specific silence of the B-FABP gene changed the proliferation and migration properties of Caki-2 cells in the same manner. The cellular property of invasion was not dependent on the B-FABP content. The diversity of the B-FABP effects on these cell-biological processes in carcinogenesis needs further elucidation.

\section{Acknowledgements}

This study was supported by grants from the European Regional Development Fund (EFRE), ProFIT grant no. 10140618. We are grateful to Silke Klotzek and Ines Baumert for excellent technical assistance.

\section{References}

1. Boord JB, Fazio S and Linton MF: Cytoplasmic fatty acid-binding proteins: emerging roles in metabolism and atherosclerosis. Curr Opin Lipidol 13: 141-147, 2002.

2. Hammamieh R, Chakraborty N, Das R and Jett M: Molecular impacts of antisense complementary to the liver fatty acid binding protein (FABP) mRNA in DU 145 prostate cancer cells in vitro. J Exp Ther Oncol 4: 195-202, 2004.

3. Hammamieh R, Chakraborty N, Barmada M, Das R and Jett M: Expression patterns of fatty acid binding proteins in breast cancer cells. J Exp Ther Oncol 5: 133-143, 2005.

4. Ohlsson G, Moreira JM, Gromov P, Sauter G and Celis JE: Loss of expression of the adipocyte-type fatty acid-binding protein (A-FABP) is associated with progression of human urothelial carcinomas. Mol Cell Proteomics 4: 570-581, 2005.

5. Mita R, Coles JE, Glubrecht DD, Sung R, Sun X and Godbout R: B-FABP-expressing radial glial cells: the malignant glioma cell of origin? Neoplasia 9: 734-744, 2007.

6. Hancke K, Grubeck D, Hauser N, Kreienberg R and Weiss JM: Adipocyte fatty acid-binding protein as a novel prognostic factor in obese breast cancer patients. Breast Cancer Res Treat 119: 367-377, 2010

7. Tölle A, Jung M, Lein M, et al: Brain-type and liver-type fatty acid-binding proteins: new tumor markers for renal cancer? BMC Cancer 9: 248, 2009.

8. Huynh HT, Larsson C, Narod S and Pollak M: Tumor suppressor activity of the gene encoding mammary-derived growth inhibitor. Cancer Res 55: 2225-2231, 1995.

9. Buhlmann C, Borchers T, Pollak M and Spener F: Fatty acid metabolism in human breast cancer cells (MCF7) transfected with heart-type fatty acid binding protein. Mol Cell Biochem 199: 41-48, 1999.
10. Fang LY, Wong TY, Chiang WF and Chen YL: Fatty-acidbinding protein 5 promotes cell proliferation and invasion in oral squamous cell carcinoma. J Oral Pathol Med 39: 342-348, 2010.

11. Goto Y, Matsuzaki Y, Kurihara S, et al: A new melanoma antigen fatty acid-binding protein 7 , involved in proliferation and invasion, is a potential target for immunotherapy and molecular target therapy. Cancer Res 66: 4443-4449, 2006.

12. Seliger B, Lichtenfels R, Atkins D, et al: Identification of fatty acid binding proteins as markers associated with the initiation and/or progression of renal cell carcinoma. Proteomics 5: 2631-2640, 2005

13. Teratani T, Domoto T, Kuriki K, et al: Detection of transcript for brain-type fatty Acid-binding protein in tumor and urine of patients with renal cell carcinoma. Urology 69: 236-240, 2007.

14. Maatman RG, van Kuppevelt TH and Veerkamp JH: Two types of fatty acid-binding protein in human kidney. Isolation, charcterization and localization. Biochem J 273 (Pt 3): 759-766, 1991.

15. Slipicevic A, Jorgensen K, Skrede M, Rosnes AK, Troen G, Davidson $B$ and Florenes VA: The fatty acid binding protein 7 (FABP7) is involved in proliferation and invasion of melanoma cells. BMC Cancer 8: 276, 2008.

16. Prinsen CF and Veerkamp JH: Transfection of L6 myoblasts with adipocyte fatty acid-binding protein cDNA does not affect fatty acid uptake but disturbs lipid metabolism and fusion. Biochem J 329 (Pt 2): 265-273, 1998.

17. Morgan EA, Forootan SS, Adamson J, et al: Expression of cutaneous fatty acid-binding protein (C-FABP) in prostate cancer: potential prognostic marker and target for tumourigenicity-suppression. Int J Oncol 32: 767-775, 2008.

18. Keler T and Sorof S: Growth promotion of transfected hepatoma cells by liver fatty acid binding protein. J Cell Physiol 157: 33-40, 1993.

19. Anthony TE, Mason HA, Gridley T, Fishell G and Heintz N: Brain lipid-binding protein is a direct target of Notch signaling in radial glial cells. Genes Dev 19: 1028-1033, 2005.

20. Bisgrove DA, Monckton EA, Packer M and Godbout R: Regulation of brain fatty acid-binding protein expression by differential phosphorylation of nuclear factor I in malignant glioma cell lines. J Biol Chem 275: 30668-30676, 2000.

21. Brun M, Coles JE, Monckton EA, Glubrecht DD, Bisgrove D and Godbout R: Nuclear factor I regulates brain fatty acid-binding protein and glial fibrillary acidic protein gene expression in malignant glioma cell lines. J Mol Biol 391: 282-300, 2009.

22. Liang Y, Bollen AW, Aldape KD and Gupta N: Nuclear FABP7 immunoreactivity is preferentially expressed in infiltrative glioma and is associated with poor prognosis in EGFR-overexpressing glioblastoma. BMC Cancer 6: 97, 2006.

23. Kaloshi G, Mokhtari K, Carpentier C, et al: FABP7 expression in glioblastomas: relation to prognosis, invasion and EGFR status. J Neurooncol 84: 245-248, 2007.

24. Haunerland NH and Spener F: Fatty acid-binding proteins insights from genetic manipulations. Prog Lipid Res 43: 328-349, 2004. 